\title{
Original process for Thymol Para-sulfonation: A Preliminary Optimization Study
}

\author{
Abderrahman Sabour 1,*, Abdelhafed El Asbahani ${ }^{1}$, Abdellah Lacherai ${ }^{1}$, Brahim El Ouadi ${ }^{2}$ \\ and Abderrahim Jilale ${ }^{1}$ \\ ${ }^{1}$ Applied Chemistry and Environment Laboratory, Team of Bio-organic Chemistry and Natural substances, \\ Faculty of Sciences, Ibn Zohr University, PoBox8106, Agadir 80000, Morocco \\ ${ }^{2}$ Laboratory of Engineering Sciences for the Environment- UMR CNRS 7356, Pole sciences et Technology, \\ Avenue Michel Crépeau 17042 La Rochelle Cedex 1, France
}

\begin{abstract}
In this study, we described an original, new, simple, and productive one-pot method for Thymol parasulfonation by using thionyl chloride $\left(\mathrm{SOCl}_{2}\right)$ as a sulfonating agent under mild conditions. Spectroscopic analysis by FT-IR, mass spectrometry, and NMR $1 \mathrm{H}$ and $13 \mathrm{C}$ confirmed the structure of the parasulfonated derivative of Thymol: the 4-hydroxy-5-isopropyl-2-methylbenzenesulfonic acid. An optimization study was carried out to perform and improve the yield of this new para-sulfonation method by studying the effect of three parameters: temperature, the molar ratio $(\eta)$ of the reactants, and solvents. The obtained results showed that the use of cyclohexane as a solvent, a molar ratio greater or equal than 3 , and working under low temperatures increase the yield of this parasulfonation considerably to reach adequately $91.3 \%$.
\end{abstract}

Keywords: Thymol; Sulfonation; Regio-selectivity; Para-Thymol sulfonic acid; Thionyle chloride; Optimization study.

\section{Introduction}

Sulfonic aromatic products are of great interest because of their many industrial applications ${ }^{1-3}$. Generally, the preparation of these products involves a sulfonation reaction. The latter is an electrophilic substitution reaction leading to the introduction of a sulfonic group $\left(-\mathrm{SO}_{3} \mathrm{H}\right)$ via a carbon-sulfur bond on the structure of an organic molecule ${ }^{4}$. It is one of the most critical reactions in industrial organic chemistry alongside nitration and chlorination ${ }^{5}$. There are many methods of sulfonation of aromatic compounds ${ }^{6}$ involving the use of sulfur trioxide, oleum, or sulfuric acid. Most of them are based on direct sulfonation, which is a reversible reaction requiring the use of sulfuric acid $\left(\mathrm{H}_{2} \mathrm{SO}_{4}\right)$ as sulfonating agent ${ }^{7}$ and working under high temperature and low pressure to be moved to the product. However, this non-mild method presents some drawbacks and limitations: i.e., poor to average yields, a decrease in selectivity, an increase in the formation of undesirable by-products and isomers, which are mainly sulfonylated compounds ${ }^{5}$ making the processes more complicated, expensive and unprofitable. Also, these conditions are dangerous on an industrial scale and unsuitable for sensitive aromatic compounds. Thymol is an aromatic phenolic compound biosynthesized naturally in some

*Corresponding author: Abderrahman Sabour

Email address: sabourabde@gmail.com

DOI: http://dx.doi.org/10.13171/mjc10602006191398as medicinal plants particularly species from Thymus and Origanum genus. It is an abundant component in many essential oils, making them highly appreciated ${ }^{8,9}$. It is purified in the form of a white crystalline substance with a strong aromatic odor. Thymol is considered among the most active natural compounds since it exhibits very important Pharmacological and biological activities such as antiseptics, antimicrobials, anti-inflammatory, analgesic, and antioxidants ones and shows consequently a promising therapeutic potential ${ }^{10-16}$. The synthesis of Thymol itself is obtained by alkylation of $\mathrm{m}$-cresol by isopropene or isopropyl alcohol using different catalyst systems ${ }^{17-24}$. Other processes have already been patented for Thymol synthesis ${ }^{25,26}$ or the synthesis of some substituted hydroxyl aromatic compounds ${ }^{27}$, and even by converting isomers of Thymol into Thymol after a sulfonation step, isomerization of the resulting sulfonic acids, heating with water and then desulfonation ${ }^{28}$.

Basing on this interest and as part of our investigation on the reactivity and improvement of the properties and reactivity of natural products, we are interested in this essential phenolic compound. We focused on this work on its sulfonation. In medicinal chemistry, sulfonation is a crucial step to

Received March 19, 2020

Accepted April 16, 2020

Published June 19, 2020 
obtain more reactive derivatives suitable for condensation reactions to give more active components. For example, with indazole derivatives, sulfonated compounds give highly biologically active sulfonamides with marked antibacterial and anticancer potential ${ }^{29}$. On the other hand, sulfonation is an option to introduce new functional groups (i.e. nitro, amino...) into the aromatic nucleus ${ }^{30}$. In the usual way, sulphuric acid is most frequently used for sulfonation of phenolic compounds ${ }^{31,32}$. This method does not give us consistent results with Thymol. So, to circumference these drawbacks, we searched to use other sulfoxide agents under mild conditions, in this case Thionyle chloride, to effect the sulfonation of Thymol. The process thus undertaken is unprecedented, proving its originality. In the light of this consideration, we have reported in this work the description and optimization of a new para-sulfonation method of Thymol. Also, the product obtained, the 4-hydroxy5-isopropyl-2-methyl benzene sulfonic or paraThymol sulfonic acid (p-TSA) is characterized by different spectroscopic techniques.

After that, we give in this work the experimental procedure of the para-sulfonation of Thymol and a performed preliminary optimization study by studying the effect of three factors: temperature, the molar ratio $\eta$ of the reactants and solvents on the yield of this original para-sulfonation reaction.

\section{Experimental section}

\subsection{General experimental procedures \\ 2.1.1. Materials and chemicals}

Thymol $(\geq 99 \%-101), \mathrm{SOCl}_{2}(\geq 99 \%)$, Petroleum Ether $\left(90 \%\right.$, pure for analysis $\left.40-60^{\circ} \mathrm{C}\right)$, n-Hexane $(\geq 99 \%)$, Dichloromethane anhydrous $(\geq 99.8 \%)$ and Chloroform $(\geq 99 \%)$ : These reagents and solvents were purchased from Sigma-Aldrich. Cyclohexane (EMSURE grade, $\geq 99.5 \%$ purity) was purchased from Merck. These products were directly used as received without further purifications. Silica gel plates (Merck, silica gel 60 GF254) were used for TLC analysis. TLC spots were detected under UV light $(\lambda \max =254 \mathrm{~nm})$.

\subsubsection{Characterization of equipment}

Fourier transform infrared spectral analyses were carried out on a Bruker Vertex 70 FT-IR spectrophotometer operated with $\mathrm{KBr}$ discs. $1 \mathrm{H}$ and 13C spectra were recorded on $400 \mathrm{MHz}$ Bruker NMR spectrometers with dimethylsulfoxide-d6 (DMSO-d6) as a solvent. The chemical shifts were expressed in ppm $(\delta)$. The GC / MS analyses were performed on an Agilent 1290 infinity equipped with a split/splitless injector, a DB-5 fused silica capillary column $(30 \mathrm{~m} \times 0,25 \mathrm{~mm} \times 0,25 \mu \mathrm{m})$ and a MassaSpectrometer detector ISQ series operating in electron impact mode at $70 \mathrm{eV}$ with mass scan range between 40 to $450 \mathrm{amu}$. This MSD was monitored by Xcalibur software (Version 3.2.1.2). The melting point was determined with an Electrothermal 9300 capillary apparatus.

\subsection{General procedure for the synthesis of p-TSA 2.2.1. Solubility tests and criteria for the choice of suitable solvents}

To carry out this reaction and to get a correct orientation to achieve the optimization study, we decided, in parallel, to perform preliminary solubility tests to choose the solvents that would be more suitable and specify the operating conditions, which would be more favorable for this reaction. The criteria that have been retained for the choice of the solvents were: i/ the reagents must be soluble therein, ii/ the final product must be insoluble, iii/ the appearance of the product and reaction time must be as short as possible. To give more chances for the formation of the product and its precipitation, we, therefore, decided first to conduct this reaction at low temperatures $\left[0^{\circ} \mathrm{C}-5^{\circ} \mathrm{C}\right]$.

We performed so firstly several solubility tests using a wide range of solvents of different polarities and properties to identify and select the suitable solvents for this reaction in which Thymol is soluble, and pTSA is insoluble. These help us to quickly and easily isolate p-TSA from the reaction mixture by vacuum filtration.

\subsubsection{Experimental protocol}

For stability reasons, this reaction was carried out at low temperatures (in an ice bath, between 0 to $5^{\circ} \mathrm{C}$ ). Thymol (13.31 mmol) was dissolved in $15 \mathrm{~mL}$ of a suitable solvent and placed in a dropping funnel. In $250 \mathrm{~mL}$ two necked round bottom flask, Thionyl chloride $\left(\mathrm{SOCl}_{2}, 39.93 \mathrm{mmol}\right)$ was dissolved in the same solvent. Thymol solution was then dropwise added to a thionyl chloride solution under stirring (500 rpm), which leads after a few minutes of contact to the beginning of the appearance of a yellow-greenish precipitate. TLC monitored this reaction for two 2 hours. The precipitate was filtered and washed three times with Cyclohexane to remove impurities and kept at room temperature in contact with a flux of ambient air inside a ventilated Laboratory extractor fume hood. There has been then a gradual change of the product color from yellow to solid white product (p-TSA) after about 15 minutes.

White solid, mp $\left(100{ }^{\circ} \mathrm{C}\right)$, Eluent: Petroleum ether/cyclohexane (3:7), Rf = 0.35; GC-MS (EI, 70 ev), m/z (\%): 230 [M+], (6), 168 (34), 152 (80), 150 (40), 136 (100), IR (KBr, cm $\left.{ }^{-1}\right): 3360(\mathrm{O}-\mathrm{H}), 1500$ and $1405(\mathrm{C}=\mathrm{C} \mathrm{Ar}), 1257$ and $1185(\mathrm{~S}=\mathrm{O}), 636(\mathrm{C}-$ S), ${ }^{1} \mathrm{H}$ NMR (DMSO-d6, $\left.300 \mathrm{MHz}, \delta, \mathrm{ppm}\right), 9.72$ (s,1H, C-OH), 7.62 (d, J = 7.8 Hz, 1H, Ar-H), 7.20 $(\mathrm{s}, \mathrm{J}=7.8 \mathrm{~Hz}, 1 \mathrm{H}, \mathrm{Ar}-\mathrm{H}), 3.12-3.10(\mathrm{~m}, \mathrm{~J}=7.0 \mathrm{~Hz}$, $1 \mathrm{H}, \mathrm{CH}), 2.70$ (s, 3H, CH3), $1.21(\mathrm{~d}, \mathrm{~J}=7.0 \mathrm{~Hz}, 6 \mathrm{H}$, $\mathrm{CH} 3) .{ }^{13} \mathrm{C}$ NMR (DMSO-d6, $100 \mathrm{MHz}, \delta, \mathrm{ppm}$ ): 160.20 (CAr), 138.21 (CAr), 138.20 (CAr), 138.17 (CHAr), 123.53 (CHAr), 117.28 (CHAr), 28.35 $(\mathrm{CH}), 23.78$ (2 x CH3), $22.53(\mathrm{CH} 3)$. 


\subsection{Optimization study}

To perform a preliminary optimization study, we suggest verifying the effect of three main parameters on the yields of this sulfonation reaction. Selected parameters for this study were: temperature, the molar ratio $(\eta)$ of the reactants, and solvent. This was be done by fixing two settings and varying the latter studied parameter. The molar ratio $(\eta)$ was taken as a ratio of the moles' number of Thionyle chloride to those of Thymol. The following formula gives it:

$\eta=\frac{n\left(\mathrm{SOCl}_{2}\right)}{n(\text { Thymol })}$

\subsubsection{Effect of temperature}

To study the effect of temperature on the sulfonation of Thymol, we used Petroleum ether as solvent and a molar ratio value equal to 3 . The same reaction was repeated three times for each of the three following temperature ranges: low temperatures (between $0^{\circ} \mathrm{C}$ and $\left.5^{\circ} \mathrm{C}\right)$, at room temperature $\left[20-25^{\circ} \mathrm{C}\right]$ and at temperatures greater or equal than $40^{\circ} \mathrm{C}$.

\subsubsection{Effect of molar ratio $(\eta)$}

In the study of the impact of molar ratio, this sulfonation reaction was carried out in petroleum ether and at low temperature $\left(0^{\circ} \mathrm{C}\right.$ to $\left.5^{\circ} \mathrm{C}\right)$. The choice of Petroleum Ether is based on the previous criteria for the solvents mentioned above. The reaction was performed for molar ratio values: 1,2 , 3 , and higher than 3 .

\subsubsection{Effect of reaction solvent}

Basing on the previous criteria related to the choice of the solvents, the same reaction was carried out at low temperatures $\left(0^{\circ} \mathrm{C}\right.$ to $\left.5^{\circ} \mathrm{C}\right)$ and for a molar ratio $\eta$ equal to 3 by using different solvents. Basing on the previous criteria, chosen solvents for this reaction were Petroleum ether, Hexane, Cyclohexane, Chloroform and Dichloromethane (DCM).

\section{Results and Discussion}

\subsection{Synthesis of $p$-TSA}

Para-sulfonation of Thymol (Scheme 1) was carried out by reaction between Thymol and thionyl chloride. Initially, solubilized Thymol in a suitable solvent (i.e. Petroleum Ether) was dropwise added at low temperature $\left(0\right.$ to $\left.5^{\circ} \mathrm{C}\right)$ to Thionyle chloride in the same solvent as for Thymol. After a few minutes, we obtained a yellow-greenish precipitate increasing depending on the duration of the reaction. After vacuum filtration and washing, the obtained yellow solid compound was kept in an aerated surface to give a white compound after contact with ambient air finally. This latter was examined by several spectral analyzes technics to verify its structure and its purity.<smiles>Cc1ccc(C(C)C)c(O)c1</smiles>

Scheme 1. Synthesis of p-TSA

After the examination of the spectral analysis (FTIR, NMR, and GC-MS) it will be clear and easy to conclude that the sulfonation of Thymol was carried out in para position related to the phenolic hydroxyl group of Thymol. Moreover, two electrophilic substitution products are possible (ortho and para). Analyzed data from FTIR, indicate clearly a specific pic of sulfonic group (wave number $(w n)=3360$ is specific to $(\mathrm{O}-\mathrm{H})$ group of Thymol, wn $=1257 \mathrm{~cm}^{-1}$ and mainly $1185 \mathrm{~cm}^{-1}$ are specific to $(\mathrm{S}=\mathrm{O})$ group, while $636 \mathrm{~cm}^{-1}$ is specific to (C-S) and as shown by NMR ${ }^{1} \mathrm{H}$ spectra, the proton peak disappears in the para position relative to the hydroxyl group. This leads us to conclude that a sulfonation reaction was carried out in the para position of Thymol. Finally, the analysis of data obtained by GC-MS confirms the purity and precisely the mass of the sulfonated product, thus synthesized. So, chemical analysis of the product obtained showed the obtaining of a single product and precisely that corresponding to para-substitution. Having only one product can be explained by the steric hindrance that promotes substitution in the para position.

\subsection{Proposed mechanism of synthesis}

To explain the reaction of Thymol with Thionyl chloride, we must take into account the two mesomeric forms of Thymol (to put in Scheme 2). Thus two electrophilic substitution products are possible (ortho and para). Chemical analysis of the product obtained showed the obtaining of a single product and precisely that corresponding to parasubstitution. Having only one product can be explained by the steric hindrance that promotes substitution in the para position. The intermediate form 5 (yellow color and unstable) undergoes oxidation by contact with the oxygen of the ambient air to give finally form 6 (white color) identified as p-TSA. 
Scheme 2 shows the mechanism proposed for this sulfonation. It is assumed that the yellow product (form 5) corresponds to para Thymol sulfoxide chloride.

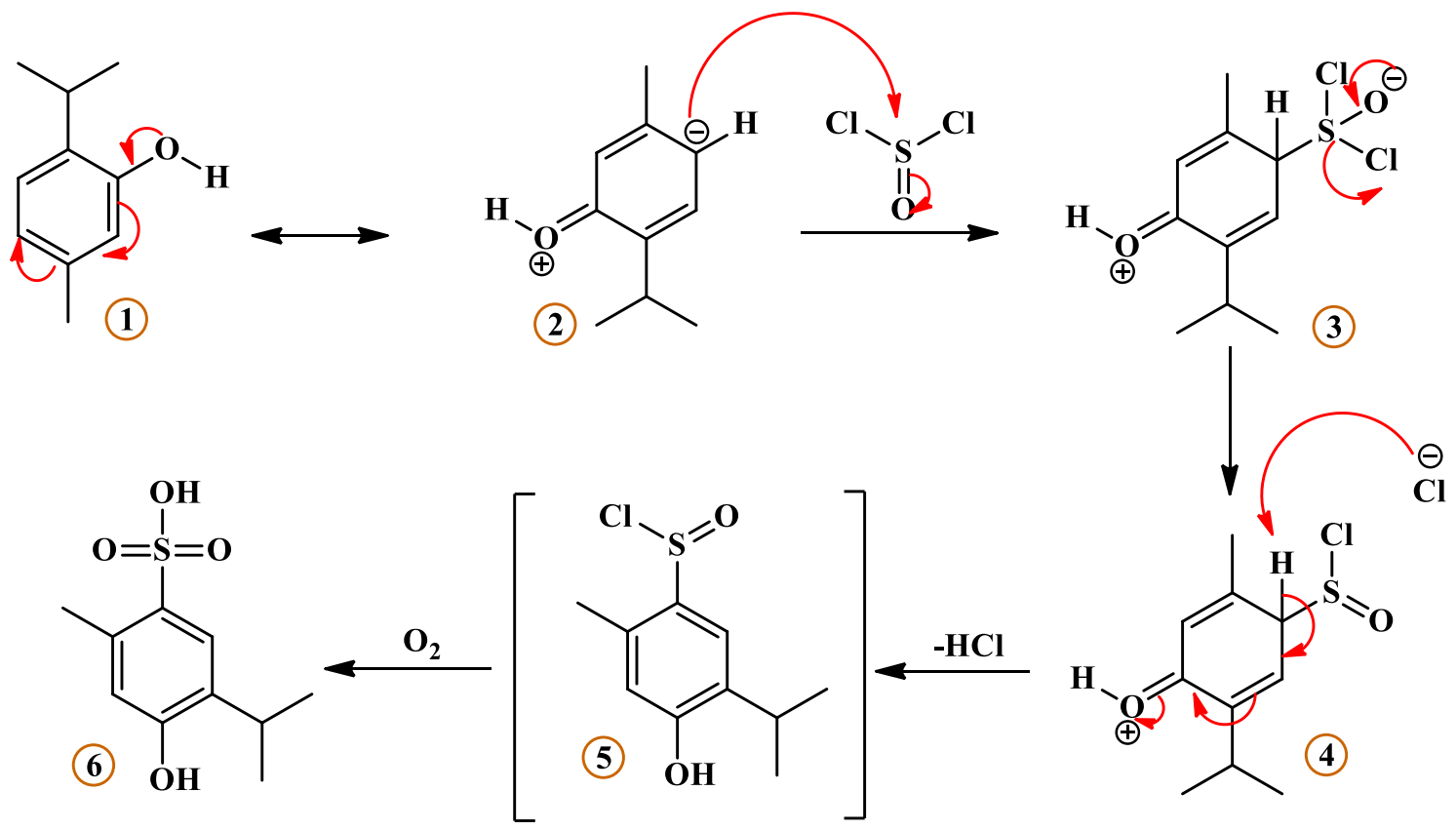

Scheme 2. Proposed reaction mechanism for Thymol sulfonation

\subsection{Optimization study}

\subsubsection{Effect of molar ratio $(\eta)$}

The study of the impact of molar ratio on this sulfonation reaction was carried out in petroleum ether and at low temperature. The choice of petroleum ether is based on the criteria of solubility of the reactants (soluble) and the final product (insoluble). Figure 2 summarizes the results obtained from average yield for three repetitions of each manipulation.

To perform this reaction at low temperatures, it is imperative to use an excess of thionyl chloride relatively to Thymol. This may increase the probabilities of the nucleophilic attack of Thymol on the sulfinic group of thionyl chloride, leading to an increase in the formation of the intermediate compound 5 (Scheme 2). More thionyl chloride is abundant in the medium; the more probabilities of the nucleophilic attack by Thymol are increased, and the sulfonation reaction is improved. Experimentally, the ratio of one molecule of Thymol to three molecules of Thionyle chloride gives the best yields for this sulfonation reaction. Still, it is useless to increase this ratio to more than three.

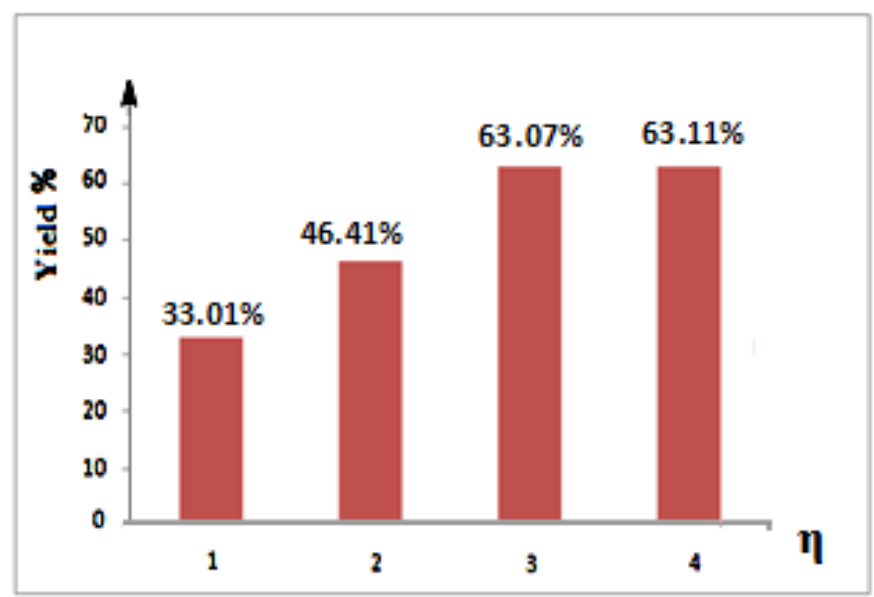

Figure 1. Effect of molar ratio on the yield of the sulfonation reaction in Petroleum Ether and at low temperature

\subsubsection{Effect of temperature}

To study the effect of temperature on the sulfonation of Thymol, we used Petroleum ether as a solvent and a molar ratio equal to 3 . The same reaction was repeated at three different temperature ranges: low temperatures (ice bath, between 0 and $5^{\circ} \mathrm{C}$ ), room temperature $\left[20-25^{\circ} \mathrm{C}\right]$, and at temperatures higher or equal to $40^{\circ} \mathrm{C}$. The reaction yields obtained are given in Table 1. The results obtained show that the low temperatures promote the sulfonation reaction with 
thionyl chloride. We note that at a temperature higher than or equal to $40^{\circ} \mathrm{C}$ the sulfonation reaction of Thymol with thionyl chloride is no longer realized. For room temperature $\left(20-25{ }^{\circ} \mathrm{C}\right)$, we notice that the reaction medium starts to release heat, and consecutively the obtained yield is $46.73 \%$. This may be explained by considering that this reaction is rather exothermic and by the instability of intermediate products at high temperatures.

Table 1. Effect of Temperature on Thymol Sulfonation in Petroleum Ether and molar ratio $\eta=3$.

\begin{tabular}{|c|c|c|c|}
\hline$\eta$ & {$\left[0-\mathbf{5}^{\circ} \mathrm{C}\right]$} & {$\left[\mathbf{2 0}-\mathbf{2 5}^{\circ} \mathrm{C}\right]$} & Higher than or equal to $\mathbf{4 0}^{\circ} \mathrm{C}$ \\
\hline 3 & 63.07 & 46.73 & No Sulfonation \\
\hline
\end{tabular}

\subsubsection{Effects of the reaction solvent}

Several tests of Thymol and p-TSA solubility were carried out to determine the solvents which respond to the already advanced criteria. Thus, the selected solvents are petroleum ether, hexane, cyclohexane, chloroform, and dichloromethane.

The solvent effect was carried out by working under optimal conditions (molar ratio $\eta=3$ and temperature [0 to $\left.5^{\circ} \mathrm{C}\right]$ ). The results obtained are collated in
Table 2. They show that the best yield is obtained for cyclohexane (91.3\%). Cyclohexane is a cyclic, symmetrical, nonpolar compound. It has a melting point close to zero, these properties and advantageous characteristics compared to other solvents, confer more stability on the product p-TSA, which is a sulfonated product susceptible to temperature.

Table 2. Obtained sulfonation yields for each solvent (molar ratio $\eta=3$ and temperature $\left[0-5^{\circ} \mathrm{C}\right]$ ).

\begin{tabular}{|c|c|}
\hline Solvents & Sulfonation yield \% \\
\hline Chloroform & 38.70 \\
\hline Cyclohexane & 91.30 \\
\hline DCM & 67.21 \\
\hline Petroleum Ether & 67.07 \\
\hline Hexane & 44.70 \\
\hline
\end{tabular}

\section{Conclusion}

4-hydroxy-5-isopropyl-2-methyl benzene sulfonic acid (p-TSA) was synthesized according to an original simple, effective, and regioselective method. It is a condensation of thionyl chloride on Thymol followed by oxidation in the ambient air. The product is characterized by different spectroscopic methods (FTIR, SM, and ${ }^{1} \mathrm{H}$ and ${ }^{13} \mathrm{C}$ NMR. This preparation has been optimized. Thus, a yield of $91 \%$ can be reached by carrying out the condensation in cyclohexane at low temperature and with a molar ratio of thionyl chloride and thymol equal to 3 . In perspective, a complementary and theoretical study is expected. This will be useful to understand best the effect of the different factors on this régioselective sulfonation and to extend it eventually to other phenolic compounds.

\section{Acknowledgments}

The authors express their gratitude and thanks to the technical staff of the Faculty of Sciences and Techniques-University La Rochelle France.

\section{References}

1- L. Jiang, L. Gao, Y. Liu, Adsorption of salicylic acid, 5-sulfosalicylic acid, and Tiron at the alumina-water interface, Colloids and Surfaces A: Physicochemical and Engineering Aspects, 2002, 211, 165-172.

2- G. Smith, U.D. Wermuth, D.J. Young, J.M. White, Polymeric structures in the metal complexes of 5-sulfosalicylic acid: The rubidium(I), cesium (I) and lead(II) analogs, Polyhedron, 2007, 26, 3645-3652.

3- Y. Shudo, M.R. Karim, R. Ohtani, M. Nakamura, $\mathrm{S}$. Hayami, Hybrids from the $\pi-\pi$ Stacking of Graphene Oxide and Aromatic Sulfonic Compounds for Improved Proton Conductivity, ChemElectroChem, 2017, 5, 238-241.

4- F. Kucera, J. Jancfi, Homogeneous and heterogeneous sulfonation of polymers: a review, Polymer Engineering \& Science, 1998, 38, 783-792.

5- O. Lindner, L. Rodefeld, Benzenesulfonic Acids, and their Derivatives, Encyclopedia of Industrial Chemistry, 2000, 5, 259-306.

6- E. Gilbert, Recent Developments in Preparative Sulfonation and Sulfation, Synthesis, 1969, 01, 3-10.

7- D. Daoust, J. Devaux, P. Godard, Mechanism and kinetics of poly (ether ether ketone) (PEEK) sulfonation in concentrated sulfuric acid at room temperature Part 1. Qualitative comparison 
between, Polymer International, 2001, 50, 917-924.

8- M. Puertas-Mejía, S. Hillebrand, E. Stashenko, P. Winterhalter, In-vitro radical scavenging activity of essential oils from Columbian plants and fractions from oregano (Origanum vulgare L) essential oil, Flavour, and Fragrance Journal, 2002, 17, 380-384.

9- L.R. Salgueiro, E, Pinto, M.J. Gonçalves, C. Pina-Vaz, C. Cavaleiro, A.G. Rodrigues, A. Palmeira, Chemical composition and antifungal activity of the essential oil of Thymbra capitata, Planta Med. 2004, 70, 572-575.

10-T. Yoshida, K. Mori, G.X. He, Inulavosin, a new thymol dimer with piscicidal activity from Inula Nervosa, Heterocycles, 1995, 41, 1923-1926.

11-F. Oke, B. Aslim, S. Oztirk, Essential oil composition, antimicrobial and antioxidant activities of Satureja cuneifolia Ten, Altundag S, Food Chem. 2009, 112, 874-879.

12-C.C. Liolios, O. Gortzi, S. Lalas., J. Tsaknis, I. Chinou, Liposomal incorporation of carvacrol and thymol isolated from the essential oil of Origanum dictamnus L. and in vitro antimicrobial activity, Food Chem. 2009, 112, 77-83.

13-N. Dirdy, L. Dubreuil, M. Pinkas, Antibacterial activity of thymol, carvacrol and cinnamaldehyde alone or in combination, Pharmazie, 1993, 48, 301-304.

14-M.G. Botelho, The minimum inhibitory concentration of oral antibacterial agents against cariogenic organisms, Microbios, 2000, 103, 31-41.

15-P. L. Teissedre, A. Waterhouse, L, Inhibition of oxidation of human low-density lipoproteins by phenolic substances in different essential oils varieties, Journal of Agricultural and Food Chemistry, 2000, 48, 3801-3805.

16-D.D. Jyoti, D. Singh, G. Kumar, M. Karnatak, S. Chandra, V.V. Prakash, R. Shankar, Thymol Chemistry: A Medicinal Toolbox, Current Bioactive Compounds, 2019, 15, 454-474.

17-S. Liu, X. Liu, C. Wang, Isopropylation of m-Cresol Catalyzed by Recoverable Acidic Ionic liquids, Industrial \& Engineering Chemistry Research, 2013, 52, 16719-16723.

18-M. Selvaraj, S. Kawi, Comparison of mesoporous and microporous solid acid catalysts for highly selective synthesis of thymol by vapor phase isopropylation of $\mathrm{m}$-cresol, Microporous and Mesoporous Materials, 2008, 109, 458-469.

19-R. Amandi, J.R. Hyde, S.K. Ross, T.J. Lotz, M. Poliakoff, Continuous reactions in supercritical fluids; a cleaner, more selective synthesis of thymol in supercritical $\mathrm{CO}_{2}$, Green Chemistry, 2005, 7, 288-293.
20-V. Umamaheswari, M. Palanichamy, V. Murugesan, Isopropylation of m-Cresol over Mesoporous Al-MCM-41 Molecular Sieves, Journal of Catalysis, 2002, 210, 367-374.

21-H. Grabowska, W. Miśta, J. Trawczyński, J. Wrzyszcz, M. Zawadzki, A method for obtaining thymol by gas phase catalytic alkylation of m-cresol over zinc aluminate spinel, Applied Catalysis A General, 2001, 220, 207-213.

22-S. Velu, S. Sivasanker, Alkylation of m-cresol with methanol and 2-propanol over calcined magnesium-aluminum hydrotalcite, Research on Chemical Intermediates, 1998, 24, 657-666.

23-C.T. O'Connor, G. Moon, W. Böhringer, J.C.Q. Fletcher, Alkylation of Phenol and m-Cresol Over Zeolites, Collection of Czechoslovak Chemical Communications, 2003, 68, 1949-1968.

24-M. Nitta, K. Yamaguchi, K. Aomura, The Alkylation of m-Cresol with Propylene by Supported Metal Sulfates, Bulletin of the Chemical Society of Japan, 1974, 47, 2897-2898.

25-P. Wimmer, H.J. Buysch, L. Puppe, Process for the preparation of thymol, United States Patent $\mathrm{N}^{\circ}$ 5,030,770, 1991.

26-P. Max, Manufacture of synthetic thymol, United States Patent $\mathrm{N}^{\circ} 1,432,298,1922$.

27-D.R. Stevens, Process for producing substituted hydroxy aromatic compounds, United States Patent $\mathrm{N}^{\circ}$ 2,603, 662, 1952.

28-R. R Bottoms, Crestwood, Production of Thymol, United States Patent $N^{\circ} 2,840,616,1958$.

29-N. Abbassi, H. Chicha, E. M. Rakib, A. Hannioui, M. Alaoui, A. Hajjaji M. Viale, Synthesis, antiproliferative and apoptotic activities of N-(6(4)-indazolyl)benzenesulfonamide derivatives as potential anticancer agents, European Journal of Medicinal Chemistry, 2012, 57, 240-249.

30-B. Oulemda, E. M. Rakib, N. Abbassi, M. Saadi, L. El Ammari, 1-(4-Methylphenylsulfonyl)-5,6dinitro-1H-indazole, Acta Crystallographica Section E Structure Reports Online, 2013, 70, 101. https://doi.org/10.1107/S1600536813034326.

31-M. Xiaoke, Li. Peng, M. Tian-Xiang, GuangWen Chu, Yong Luo, Hai-Kui Zou, Study on phenol sulfonation by concentrated sulfuric acid: Kinetics and process optimization, Chemical Engineering Science, 2019, 202, 15-25.

32-A. Abdolmaleki, M. Zhiani, M. Maleki, S. Borandeh, K. Firouz, Preparation and evaluation of sulfonated polyoxadiazole membrane containing phenol moiety for PEMFC application, Polymer, 2015, 75, 17-24. 